\title{
ANÁLISE DA VIABILIDADE ECONÔMICA DA FIBRA ORGÂNICA PARA CONTENÇÃO DE HIDROCARBONETOS
}

\author{
Jessica Syrio Callefi ${ }^{1}$, Walder Taidi Vitor Kuroda ${ }^{1}$ \\ ${ }^{1}$ Universidade Estadual de Maringá, 8720-900, Maringá, Brasil.
}

E-mail: jessica.callefi@gmail.com

Recebido em: 15/05/2018

Aceito em: 13/07/2018

\section{RESUMO}

Diversas empresas criam novas soluções em produtos no intuito de melhorar a coleta e destinação de resíduos. Uma destas novas soluções é a substituição da serragem pelo uso da fibra orgânica para a contenção do derramamento de óleo. Assim, este artigo tem o objetivo de avaliar a viabilidade econômica do uso da fibra orgânica ao invés da serragem. Foram utilizados: Fluxo de Caixa, Payback, Valor Presente Líquido (VPL) e Taxa Interna de Retorno (TIR) para a análise da viabilidade econômica. A metodologia utilizada foi a do estudo de caso. Como resultados, observa-se que a fibra orgânica de alta absorção é viável economicamente, pois, ainda que seu custo inicial de obtenção seja mais alto, este se torna mais barato no custo do descarte.

Palavras-chave: Fibra Orgânica. Serragem. Viabilidade Econômica. Contenção de hidrocarbonetos.

\section{Introdução}

A competitividade das empresas pode ser prejudicial para o meio ambiente e para a sociedade quando as ações não são pensadas de maneira sustentável [1]. Os óleos lubrificantes são produzidos em grande quantidade em vista de atender a demanda industrial que necessita desta matéria prima para seu funcionamento. Entretanto, estes óleos possuem características nocivas ao meio ambiente, por possuírem metais pesados, os quais contaminam os lençóis freáticos e os rios, e, por sobrenadarem os rios, lagos e mares, o que impede a fauna e flora aquática de se desenvolverem [2]. Além disso, os óleos lubrificantes, em geral, possuem diversas substâncias como o cádmio, o chumbo e o arsênio, que são bioacumulativos e podem se tornar tóxicos para os seres vivos [3].

Dessa forma, estes óleos formados de hidrocarbonetos devem receber uma destinação correta para evitar maiores danos ao meio ambiente. A solução mais usual para conter o derramamento de óleo hidrocarboneto, em pequenas quantidades, nas indústrias é a serragem, a qual é um resíduo advindo do corte da madeira. Porém, novos produtos como a fibra orgânica vêm ganhando espaço no mercado, porque estes materiais incorporam o hidrocarboneto à sua massa, e assim, realizam o processo de absorção ou de adsorção [4].

Estudos nacionais [2,5] e internacionais [6-9] têm buscado soluções para a retenção de óleos utilizados em indústrias. Estes estudos testaram diversos tipos de materiais como: celulose, argilas e filtros fabricados com materiais orgânicos, no intuito de analisar o desempenho deles para encontrar alternativas eficientes e de baixo custo.

Sabe-se que, quanto maior a superfície externa de um sólido com capacidade de adsorção, maior será a quantidade de hidrocarbonetos retidos [10]. Tanto os processos de adsorção quanto absorção estão sujeitos às leis de atração física, pelas forças intermoleculares fracas e químicas pelas interações moleculares fortes por ligações covalentes [10]. Porém, após a serragem ou a fibra orgânica entrarem em contato com o hidrocarboneto, tornam-se contaminantes secundários de classe I, e assim, necessitam de uma destinação diferente [11].

A partir da compreensão da importância ambiental deste tipo de material e a sua utilização em larga escala no contexto atual, objetivou-se neste artigo discutir sobre a viabilidade entre a utilização da serragem ou da fibra orgânica neste processo de contenção de hidrocarbonetos.

A fibra orgânica utilizada para a pesquisa advém de resíduos de raspas de couro bovino, a qual passa por um processo que retira seus elementos polares, tornando-a apolar, o que auxilia em uma maior absorção de hidrocarbonetos.

Entende-se que a questão da viabilidade econômica também é importante, uma vez que as empresas tendem a investir na questão ambiental por força de lei, primeiramente. E assim, as empresas tendem a buscar alternativas que sejam as menos dispendiosas possíveis na questão financeira.

Com esse entendimento, utilizou-se de ferramentas financeiras para realizar a análise da viabilidade econômica de uma empresa em utilizar serragem ou fibra orgânica na contenção dos derramamentos de óleo. Já que a viabilidade financeira de um produto tem o intuito de estimar o desempenho financeiro resultante da aplicação do mesmo [12], possibilitando o uso racionalizado do recurso do capital [13]. Ademais, as decisões de investimentos são essenciais para o sucesso da empresa, pois asseguram a potencialização de lucro dos acionistas [14]. 
Nesta pesquisa foram utilizados: Fluxo de Caixa, Payback, Valor Presente Líquido (VPL) e Taxa Interna de Retorno (TIR).

O Fluxo de Caixa pode ser compreendido como uma ferramenta de controle financeiro que auxilia os gestores no foco principal do projeto e auxilia nas tomadas de decisões [15]. Sua representação é dada por um gráfico com a linha temporal dos resultados do fluxo de caixa [14].

O Payback é um índice financeiro utilizado para calcular o tempo de retorno total sobre o investimento [16]. Ademais, o Payback é utilizado como um indicativo de risco para o investimento financeiro, apesar de não considerar o valor do dinheiro no tempo [14]. A Equação 1 aponta o tempo de retorno do investimento.

$$
I=\sum_{t=1}^{T} \frac{F C_{t}}{(1+K)^{t}}
$$

Fonte: [17].

Onde, I representa o investimento inicial, FC o fluxo de caixa no período, $\mathrm{K}$ o custo do capital, e $\mathrm{T}$ o tempo do retorno do investimento [12].

O Valor Presente Líquido (VPL) traz o valor atual do investimento para um valor futuro, com o objetivo de representar a diferença entre o valor presente das entradas e o valor presente das saídas de caixa [16]. Este cálculo é dado pela Equação 2 e entende-se que o VPL deve ser maior que zero para que seja economicamente viável [12].

$$
V P L=-I+\sum_{t=1}^{n} \frac{F C_{t}}{(1+K)^{t}}
$$

Fonte: [17]

$\mathrm{Na}$ Equação 2, I se refere ao investimento inicial, o $\sum$ (somatório) trata da soma das datas do período 1 até n, FC representa o fluxo de caixa no t-ésimo período e $\mathrm{K}$ é referente ao custo do capital [17].

Por fim, a Taxa Interna de Retorno (TIR) também é uma ferramenta de avaliação de investimento, que consiste em avaliar as propostas de investimentos igualando os fluxos de entrada com os de saída de caixa $[14,16]$.

O TIR pode ser calculado a partir da Equação 3, onde o VPL é o Valor Presente Líquido calculado com a Equação 2, I representa o investimento inicial e Fc o fluxo de caixa no período [17].

$$
V P L=-I+\sum_{\substack{t=1 \\ \text { Fonte: [17]. }}}^{n=N} \frac{F c_{t}}{(1+T i r)^{t}}=0
$$

Quanto maior for a taxa resultante do cálculo, melhor será o retorno do investimento do projeto [17].

A partir destes critérios é possível que a empresa verifique se deve ou não investir em um determinado projeto, ou no caso desta pesquisa, em um novo produto, com dados claros e robustos.

\section{Metodologia}

A metodologia utilizada para a análise deste estudo é a do estudo de caso [18]. Pois, conforme apresentado por [19], o estudo de caso é uma pesquisa de natureza empírica que examina um determinado fenômeno, dentro de um contexto específico.

$\mathrm{O}$ estudo foi dividido em duas partes, a primeira de observação e coleta de dados e a segunda de análise. Para a primeira etapa, elegeu-se uma oficina de caminhões que produz um grande volume de resíduos de hidrocarbonetos.

Nesta empresa, coletaram-se os dados referentes à demanda de resíduo descartado por mês com os próprios gerentes da empresa. Após, realizou-se um ensaio com a fibra orgânica de alta absorção e com a serragem, com o objetivo de avaliar quanto cada material poderia absorver de óleo. Nesta parte do experimento, explicou-se para os colaboradores da empresa como aplicar a fibra orgânica sobre o óleo derramado e como coletá-lo. O mesmo sucedeu com a serragem. O procedimento consistia em jogar uma quantidade de pó de serra ou de fibra orgânica, a qual o colaborador julgasse necessária em cima da poça de óleo que se desejava conter. Após, este material fora varrido e pesado. As informações obtidas foram anotadas e são apresentadas na sequência.

\subsection{Coleta de dados}

Coletaram-se os dados da serragem e da fibra orgânica de alta absorção separadamente. Nas Tabelas 1 e 2, N representa a sequência dos dados coletados, $\mathrm{T}$ o tempo necessário em segundos para aplicar a serragem ou fibra orgânica e limpar a poça de óleo.

A quantidade de absorvente (Qa) utilizada foi pesada, bem como a quantidade de resíduos (Qr). Já a quantidade de hidrocarboneto absorvido ( $Q$ h) foi obtida através da diferença entre Qr e Qa. Calculou-se também a porcentagem de óleo retido pela quantidade de absorvente utilizada (\%Oa) e a quantidade de óleo retido pela massa total do resíduo $(\% \mathrm{Om})$. 
Tabela 1 - Coleta de dados da serragem.

\begin{tabular}{ccccccc}
\hline $\mathbf{N}$ & $\mathbf{T}(\mathbf{s})$ & Qa (g) & Qr(g) & Qh (g) & \%Oa & $\begin{array}{c}\text { \% } \\
\text { Om }\end{array}$ \\
\hline 1 & 20 & 68 & 81 & 13 & 19,1 & 16,0 \\
2 & 20 & 95 & 113 & 18 & 18,9 & 15,9 \\
3 & 20 & 79 & 94 & 15 & 19,0 & 16,0 \\
4 & 23 & 102 & 122 & 20 & 19,6 & 16,4 \\
5 & 25 & 130 & 155 & 25 & 19,2 & 16,1 \\
\hline
\end{tabular}

Tabela 2 - Coleta da Fibra orgânica.

\begin{tabular}{ccccccc}
\hline $\mathbf{N}$ & $\mathbf{T}(\mathbf{s})$ & $\mathbf{Q a}(\mathbf{g})$ & $\mathbf{Q r}(\mathbf{g})$ & $\mathbf{Q h}(\mathbf{g})$ & $\mathbf{\% O a}$ & $\begin{array}{c}\% \\
\mathbf{O m}\end{array}$ \\
\hline 1 & 30 & 9 & 28 & 19 & 211,1 & 67,9 \\
2 & 35 & 8 & 23 & 15 & 187,5 & 65,2 \\
3 & 50 & 15 & 44 & 29 & 193,3 & 65,9 \\
4 & 20 & 5 & 16 & 11 & 220,0 & 68,8 \\
5 & 30 & 11 & 33 & 22 & 200,0 & 66,7 \\
\hline
\end{tabular}

A empresa, objeto do estudo de caso, possui contrato com uma empresa terceirizada especializada no descarte do resíduo contaminado grau 1 . As coletas de resíduos acontecem a cada 15 dias. A quantidade em quilogramas de resíduo descartado nos últimos 5 meses está descrita na Tabela 3.

Tabela 3 - Descarte do resíduo classe 1.

\begin{tabular}{cc}
\hline Meses $/ \mathbf{2 0 1 7}$ & Quantidade de resíduo descartado (kg) \\
\hline Abril & 11020 \\
Maio & 10150 \\
Junho & 11030 \\
Julho & 10370 \\
Agosto & 11400 \\
\hline
\end{tabular}

Outros dados ainda são relevantes para realizar a análise. A fibra orgânica possui o valor de $\mathrm{R} \$ 9,89$ por quilograma. A serragem é obtida de madeireiras próximas à oficina e seu valor é de $\mathrm{R} \$ 0,20$ por quilograma. Além disso, o valor do descarte de resíduo é de $\mathrm{R} \$ 1,00$ por quilograma de resíduo contaminado, tanto para a serragem quanto para a fibra orgânica.

\section{Resultados e discussões}

\subsection{Análise dos resultados}

A partir das tabelas 1 e 2, observa-se que são necessários, em média 22 segundos para a limpeza de uma poça de óleo com a serragem contaminada e 33 segundos para a limpeza com o uso de fibra orgânica contaminada. Em razão da técnica de uso, o tempo de limpeza da fibra orgânica é maior em $33 \%$ em relação ao tempo de limpeza da serragem. Porém, em relação ao salário do trabalhador, este fator não é relevante, uma vez que o tempo de limpeza não onera o tempo de trabalho do colaborador de maneira significativa.

Sobre a absorção de hidrocarbonetos, verifica-se que a porcentagem de óleo retido pela quantidade de serragem utilizada é, em média, 19,2\% enquanto a porcentagem de óleo retido pela quantidade de fibra utilizada é de $202,4 \%$. Portanto, a fibra absorve em torno de duas vezes a sua massa, enquanto a serragem absorve menos de $20 \%$ de sua massa. Esta informação contrasta com a embalagem do produto, a qual afirmava uma absorção de 6 a 8 vezes a sua massa, diferentemente do resultado obtido no teste.

Com os dados da Tabela 1, tem-se que a média de óleo hidrocarboneto retido no resíduo gerado com o uso de serragem é de $16 \%$. Em posse deste dado, utilizou-se este valor para estimar a quantidade de hidrocarboneto presente no resíduo descartado por mês, conforme apresentado pela Tabela 4. A quantidade de serragem foi obtida através da diferença entre a quantidade de resíduo total diminuído da quantidade de hidrocarboneto.

Tabela 4 - Quantidade de hidrocarboneto e absorvente no descarte da serragem.

\begin{tabular}{cccc}
\hline $\begin{array}{c}\text { Meses/ } \\
\mathbf{2 0 1 7}\end{array}$ & $\begin{array}{c}\text { Quantidade de } \\
\text { resíduo com } \\
\text { Serragem }(\mathbf{k g})\end{array}$ & Hidrocarboneto $\mathbf{( k g )}$ & $\begin{array}{c}\text { Serragem } \\
(\mathbf{k g})\end{array}$ \\
\hline Abril & 11020 & 1773 & 9247 \\
Maio & 10150 & 1634 & 8516 \\
Junho & 11030 & 1775 & 9255 \\
Julho & 10370 & 1669 & 8701 \\
Agosto & 11400 & 1835 & 9565 \\
\hline
\end{tabular}

Considerando, a partir do experimento realizado, que a fibra orgânica absorve duas vezes a sua massa, analisou-se o quanto de fibra orgânica seria necessária por mês para absorver o hidrocarboneto presente no resíduo descartado e quanto seria gerado de resíduo neste novo processo, com a utilização da fibra orgânica ao invés da serragem. Os resultados estão apresentados na Tabela 5.

Tabela 5-Quantidade de hidrocarboneto e absorvente no descarte.

\begin{tabular}{cccc}
\hline Meses/2017 & $\begin{array}{c}\text { Quantidade de } \\
\text { Hidrocarboneto (kg) }\end{array}$ & Fibra orgânica (kg) & $\begin{array}{c}\text { Resíduo com a } \\
\text { fibra (kg) }\end{array}$ \\
\hline Abril & 1773 & 886,5 & 2659,5 \\
Maio & 1634 & 817 & 2451 \\
Junho & 1775 & 887,5 & 2662,5 \\
Julho & 1669 & 834,5 & 2503,5 \\
Agosto & 1835 & 917,5 & 2752,5 \\
\hline
\end{tabular}

Como a serragem e a fibra orgânica não necessitam de um investimento inicial prévio em equipamentos e instalações e o valor pago na mão de obra ser o mesmo tanto para a serragem quanto para a coleta do resíduo com a fibra orgânica, entende-se que o custo será dado somente pelo custo da obtenção da matéria prima e o custo pela destinação correta do resíduo. Assim, elaboraram-se as Tabela 6 e 7 que apresentam os custos da serragem e fibra orgânica respectivamente.

Os dados mostram que a fibra orgânica de alta absorção apresenta maior vantagem em relação ao custo total. Verificouse que, mesmo que exista um gasto maior na aquisição da fibra orgânica em relação à serragem, observa-se que a fibra orgânica se torna viável quando se analisa o custo do descarte total dos resíduos. 
Tabela 6 - Custo da Serragem.

\begin{tabular}{cccccc}
\hline Meses/2017 & $\begin{array}{c}\text { Quantidade de } \\
\text { Serragem }(\mathbf{k g})\end{array}$ & Custo de aquisição (R\$) & $\begin{array}{c}\text { Quantidade de resíduo com } \\
\text { Serragem (kg) }\end{array}$ & Custo do descarte (R\$) & Custo total (R\$) \\
\hline Abril & 9247 & 1849,4 & 11020 & 11020 & 12869,4 \\
Maio & 8516 & 1703,2 & 10150 & 10150 & 11853,2 \\
Junho & 9255 & 1851 & 11030 & 11030 & 10370 \\
Julho & 8701 & 1740,2 & 10370 & 11400 & 12881 \\
Agosto & 9565 & 1913 & 11400 & & 53970 \\
\hline TOTAL & & 9056,8 & & & 53026,8 \\
\hline
\end{tabular}

Tabela 7 - Custo da Fibra Orgânica.

\begin{tabular}{cccccc}
\hline Meses/2017 & $\begin{array}{c}\text { Quantidade de } \\
\text { Fibra Orgânica } \\
(\mathbf{k g})\end{array}$ & Custo de aquisição (R\$) & $\begin{array}{c}\text { Quantidade de resíduo com } \\
\text { a Fibra Orgânica (kg) }\end{array}$ & Custo do descarte (R\$) & Custo total (R\$) \\
\hline Abril & 886,5 & 8767,49 & 2659,5 & 2659,5 \\
Maio & 817 & 8080,13 & 2451 & 2451 & 11426,99 \\
Junho & 887,5 & 8777,38 & 2662,5 & 2662,5 & 10531,13 \\
Julho & 834,5 & 8253,21 & 2503,5 & 2503,5 & 11439,88 \\
Agosto & 917,5 & 9074,08 & 2752,5 & 2752,5 & 10756,71 \\
\hline TOTAL & & 42952,27 & & \multicolumn{2}{c}{13029} \\
\hline
\end{tabular}

\subsection{Análise de investimento}

No intuito de melhorar a robustez dos dados, decidiu-se por realizar a análise financeira do investimento para comparar quais seriam os ganhos em longo prazo com a utilização da fibra orgânica. Primeiramente, realizou-se a análise do Fluxo de Caixa. $\mathrm{O}$ investimento do capital de giro no primeiro mês é dado

Tabela 8 - Fluxo de Caixa entre a serragem e a fibra orgânica

\begin{tabular}{|c|c|c|c|c|c|c|}
\hline Tempo (meses) & $\mathbf{0}$ & 1 & 2 & 3 & 4 & 5 \\
\hline $\begin{array}{c}\text { Investimento Capital de } \\
\text { Giro }\end{array}$ & $-6918,09$ & & & & & \\
\hline \multicolumn{7}{|l|}{ Serragem } \\
\hline Custos Serragem & & 1849,40 & 1703,20 & 1851,00 & 1740,20 & 1913,00 \\
\hline Descarte & & 11020,00 & 10150,00 & 11030,00 & 10370,00 & 11400,00 \\
\hline Subtotal & & 12869,40 & 11853,20 & 12881,00 & 12110,20 & 13313,00 \\
\hline \multicolumn{7}{|l|}{ Fibra Orgânica } \\
\hline Custo Fibra Orgânica & & 8767,49 & 8080,13 & 8777,38 & 8253,21 & 9074,08 \\
\hline Descarte & & 2659,50 & 2451,00 & 2662,50 & 2503,50 & 2752,50 \\
\hline Subtotal & & 11426,99 & 10531,13 & 11439,88 & 10756,71 & 11826,58 \\
\hline FC Líquido & $\begin{array}{l}-6918,09 \\
\end{array}$ & 1442,42 & 1322,07 & 1441,13 & 1353,50 & 1486,43 \\
\hline
\end{tabular}

pelo valor total do custo da fibra orgânica, diminuído do valor total da serragem. Desta forma, $\mathrm{R} \$ 6.918,09$ deveriam ser desembolsados, inicialmente, para se utilizar a fibra orgânica ao invés da serragem. Os valores mês a mês são apresentados na Tabela 8. 
A partir dos dados calculados, realizou-se o cálculo do Payback, utilizando a Equação 1. Os resultados relativos ao cálculo de cada mês estão apresentados na Tabela 9.

Tabela 9-Payback para o cálculo do retorno do investimento

\begin{tabular}{ccc}
\hline $\mathbf{N}$ & Fluxo de Caixa & $\begin{array}{c}\text { Fluxo de Caixa } \\
\text { Líquido }\end{array}$ \\
\hline 0 & $-6918,09$ & $-6918,09$ \\
1 & $-5475,67$ & 1442,42 \\
2 & $-4153,60$ & 1322,07 \\
3 & $-2712,48$ & 1441,13 \\
4 & $-1358,98$ & 1353,50 \\
5 & 127,44 & 1486,43 \\
\hline
\end{tabular}

De acordo com a Tabela 9, verifica-se que o valor poupado por mês com o uso da fibra orgânica no lugar da serragem é, em média, R\$1.409,11. Dessa forma, é possível obter o retorno do investimento dentro de cinco meses.

Outro fator a ser calculado dentro da análise de viabilidade é o Valor Presente Líquido (VPL). Considerando que a taxa atratividade deva ser maior que a taxa de juros da poupança, a qual utiliza-se $0,5 \%$ ao mês e utilizando os dados do Fluxo de Caixa já obtidos pelo Quadro 1, calculou-se o VPL do uso da fibra orgânica de alta absorção utilizando a Equação 2.

Deste cálculo, obteve-se o valor do VPL de 22,4. Como o valor do VPL é positivo, sabe-se que este investimento é viável.

Por fim, calculou-se a Taxa de Retorno Interna (TIR) utilizando a Equação 3. Obteve-se o valor do TIR de 0,608\%. Nota se que o valor obtido da TIR é considerado um valor ligeiramente acima da taxa de investimento da poupança. Portanto, mais atrativo.

\section{Conclusões}

A partir do estudo realizado, conclui-se que a fibra orgânicé viável em relação à serragem, mesmo com seu valor inicial de aquisição mais elevado. Sendo este fator superado pelo custo do descarte que é maior.

Observou-se que existia uma diferença em relação às informações da embalagem e aos testes realizados. Este fator pode ter sido influenciado pela falta de preparo dos colaboradores em utilizar a fibra orgânica. Porém, ainda assim, este produto é mais interessante do que a serragem.

Outro ponto relevante sobre os testes é a possibilidade de utilizar a massa do resíduo em sua total capacidade, uma vez que a primeira utilização da fibra orgânica a tornava muito encharcada, mas a segunda utilização continha partes secas, as quais poderiam ser utilizadas novamente na contenção de outras poças de óleo. Ademais, o óleo recolhido a partir da absorção pela fibra orgânica poderia ser reaproveitado se utilizado algum processo, como o auxílio de uma prensagem, que pudesse extrair este óleo para a reutilização do mesmo.

Além disso, os estudos desta pesquisa mostram que novas soluções podem ser implementadas para auxiliar na responsabilidade ambiental das empresas e ainda assim serem menos dispendiosas.

\section{ANALYSIS OF THE ECONOMIC VIABILITY OF ORGANIC FIBER FOR HYDROCARBON CONTENT}

ABSTRACT: Several companies create new product solutions in order to improve the waste collection and disposal. One of these new solutions is the replacement of sawdust by using organic fiber to contain the oil spill. Therefore, this article aims at evaluating the economic viability of using organic fiber instead of sawdust. Cash Flow, Payback, Net Present Value (NPV) and the Internal Rate of Return (IRR) were used to analyze the economic viability. A study case was used as methodology. The results showed that the high absorption organic fiber is economically viable, since even if the initial cost for obtaining it is high, it becomes cheaper when considering its disposal cost.

Keywords: Sawdust. Organic fiber. Economic viability. High absorption.

\section{Referências}

[1] LEITE, P. R. Logística reversa: meio ambiente e competitividade. São Paulo: Prentice-Hall, 2005.

[2] MUNIZ, I. C.; BRAGA, R. M. Q. L. O gerenciamento de Óleos Lubrificantes Usados ou Contaminados e Suas Embalagens: Estudo de Caso de Uma Empresa de Logística na Região Norte do Brasil. Sistema e Gestão, Vol. 1, n. 1, 2015.

[3] SOHN, H. Gerenciamento de Óleos Lubrificantes Usados ou Contaminados Guia Básico. Associação de Proteção ao Meio Ambiente de Cianorte APROMAC, 2005

[4] THE INTERNATIONAL TANKER OWNERS POLLUTION FEDERATION LIMITED - ITOPF. Use of Sorbent Materials in Oil Spill Response. 2014. Disponível em: http://www.itopf.com/knowledge-resources/documentsguides/document/tip-8-use-of-sorbent-materials-in-oil-spill-response/>. Acesso em 22 de maio 2017.

[5] MAGALHÃES, M.A.; MONACO, P. A. V. L.; MATOS, A. T. D. Uso de filtros orgânicos na remoção de óleos e graxas presentes na água residuária de suinocultura. Engenharia na Agricultura, Vol. 21, n. 4, p. 387-395, 2013.

[6] GITIPOUR, S.; HOSSEINPOUR, M.A.; HEIDARZADEH, N.; YOUSEFI, P.; FATHOLLAHI, A. Application of Modified Clays in Geosynthetic Clay Liners for Containment of Petroleum Contaminated Sites. International Journal of Environmental Research, Vol. 9, n.1, p. 317-322, 2015.

[7] URUM, K.; GRIGSON, S.; PEKDEMIR, T.; MCMENAMY, S. A comparison of the efficiency of different surfactants for removal of crude oil from contaminated soils. Chemosphere, 62, 1403-1410, 2005.

[8]AMIN, J.S.; ABKENAR, M.V.; ZENDEHBOUDI, S. Natural Sorbent for Oil Spill Cleanup from Water Surface: Environmental Implication. Industrial \& engineering chemistry research, Vol. 54, n. 43, p. 10615-10621, 2015. 
[9] TURSIA, A.; BENEDUCI, A.; CHIDICHIMO, F.; VIETRO, N.; CHIDICHIMO, G. Remediation of hydrocarbons polluted water by hydrophobic functionalized cellulose. Chemosphere, Vol. 201, n. 1, p. 530-539, 2018

[10] GOMIDE, R. Operações Unitárias. São Paulo: Eterg Engenharia, 1980.

[11] ASSOCIAÇÃO BRASILEIRA DE NORMAS TÉCNICAS. Classificação de Resíduos Sólidos: NBR 10.004. São Paulo, 2004

[12] RODRIGUES, K. F. C.; ROZENFELD, H., Análise de Viabilidade Econômica, 2015. Disponível em: http://www.portaldeconhecimentos.org.br Acesso em: 19 de maio 2017.

[13] CASAROTTO FILHO, N.; KOPITTKE, B. H. Análise de Investimentos: matemática financeira, engenharia econômica, tomada de decisão, estratégia empresarial. 9 ed. São Paulo: Atlas, 2006.

[14] LEMES, A. B. J.; RIGO, C. M.; CHEROBIM, A. M. S. Administração financeira: princípios, fundamentos e pratica brasileira. Rio de Janeiro: Campus, 2002.

[15] GITMAN, L. J. Princípios de administração financeira. 12 ed. São Paulo: Pearson, 2010

[16] KASSAI, J. R. et al. Retorno de investimento: abordagem matemática e contábil do lucro empresarial. 3 ed. São Paulo: Atlas, 2014.

[17] SAMANEZ, C. P. Gestão de Investimentos e Geração de Valor. São Paulo: Pearson Prentice Hall, 2007.

[18] YIN, R. K. Estudo de caso: planejamento e métodos. $3^{\mathrm{a}}$ ed. Porto Alegre: Bookman, 2007.

[19] MIGUEL, P. A. C. Estudo de caso na engenharia de produção: estruturação e recomendações para sua condução. Produção, Vol. 17, n. 1, p. 216-229, 2007. 